\title{
Guided self-help concreteness training as an intervention for major depression in primary care: a Phase II randomized controlled trial
}

\author{
E. R. Watkins ${ }^{1 *}$, R. S. Taylor ${ }^{2}$, R. Byng ${ }^{3}$, C. Baeyens ${ }^{1}$, R. Read $^{1}$, K. Pearson ${ }^{1}$ and L. Watson ${ }^{1}$ \\ ${ }^{1}$ Mood Disorders Centre, University of Exeter, UK \\ ${ }^{2}$ Peninsula Medical School, University of Exeter, UK \\ ${ }^{3}$ Peninsula Medical School, University of Plymouth, UK
}

Background. The development of widely accessible, effective psychological interventions for depression is a priority. This randomized trial provides the first controlled data on an innovative cognitive bias modification (CBM) training guided self-help intervention for depression.

\begin{abstract}
Method. One hundred and twenty-one consecutively recruited participants meeting criteria for current major depression were randomly allocated to treatment as usual (TAU) or to TAU plus concreteness training (CNT) guided self-help or to TAU plus relaxation training (RT) guided self-help. CNT involved repeated practice at mental exercises designed to switch patients from an unhelpful abstract thinking habit to a helpful concrete thinking habit, thereby targeting depressogenic cognitive processes (rumination, overgeneralization).

Results. The addition of CNT to TAU significantly improved depressive symptoms at post-treatment [mean difference on the Hamilton Rating Scale for Depression (HAMD) 4.28, 95\% confidence interval (CI) 1.29-7.26], 3- and 6-month follow-ups, and for rumination and overgeneralization post-treatment. There was no difference in the reduction of symptoms between CNT and RT (mean difference on the HAMD 1.98, 95\% CI -1.14 to 5.11), although CNT significantly reduced rumination and overgeneralization relative to RT post-treatment, suggesting a specific benefit on these cognitive processes.
\end{abstract}

Conclusions. This study provides preliminary evidence that CNT guided self-help may be a useful addition to TAU in treating major depression in primary care, although the effect was not significantly different from an existing active treatment (RT) matched for structural and common factors. Because of its relative brevity and distinct format, it may have value as an additional innovative approach to increase the accessibility of treatment choices for depression.

Received 28 January 2011; Revised 29 September 2011; Accepted 30 September 2011; First published online 16 November 2011

Key words : Cognitive training, depression, guided self-help, randomized controlled trial, rumination.

\section{Introduction}

Depression is a prevalent, chronic and recurrent disorder (Blazer et al. 1994; Judd, 1997), with severe costs for the individual, including impaired social and occupational functioning, ill health, increased mortality and suicide (Wulsin et al. 2004), and major economic costs for society (Layard, 2005; Ustun et al. 2004). Although randomized controlled trials (RCTs) indicate that psychological treatments (e.g. cognitive behavioural therapy, CBT) are efficacious for depression, its high prevalence means that other approaches are required to supplement individual psychotherapy

* Address for correspondence: Professor E. R. Watkins, Mood Disorders Centre, School of Psychology, University of Exeter, Exeter EX4 4Q, UK.

(Email : e.r.watkins@exeter.ac.uk) to reduce overall depression morbidity. The development of non-traditional delivery systems has been recommended to optimize the accessibility of evidence-based interventions for all patients (Hollon et al. 2002; Layard, 2005).

Non-traditional delivery systems include online computerized CBT programmes (Christensen et al. 2004), therapist-delivered internet CBT using instant messaging (Kessler et al. 2009) and guided self-help interventions (Gellatly et al. 2007), in which a trained worker supports the patient in implementing a selfhelp package, typically bibliotherapy. Such approaches are a key element within the low-intensity component of the Improving Access to Psychological Treatment (IAPT) initiative in the UK, and often involve variants of CBT that emphasize psychoeducation, require reduced therapist contact and/or can be delivered without face-to-face meetings. 
An alternative non-traditional treatment approach is to directly modify the psychological processes hypothesized to be involved in the maintenance of depression. This cognitive bias modification (CBM) training approach uses repeated practice on appropriate cognitive tasks to train participants to respond in a way that is either consistent or inconsistent with an identified bias (e.g. attentional bias towards negative information). This approach has demonstrated the modifiability of cognitive biases and their causal role in the maintenance of anxious (e.g. MacLeod et al. 2002; Mathews \& MacLeod, 2002) and depressed mood (Watkins et al. 2008), with recent evidence that repeated CBM training to reduce negative attentional bias successfully reduces anxiety and depression (Hakamata et al. 2010; Wells \& Beevers, 2010). The CBM approach is potentially a significant treatment innovation as it directly targets processes implicated in psychopathology through repeated training, whereas established psychotherapies only indirectly influence cognitive processes through talking and behavioural plans. Moreover, CBM interventions can be delivered with reduced therapist contact, reducing their cost, and increasing their accessibility.

This study reports the first exploratory RCT of an innovative guided self-help intervention using CBM training focused on reducing two important cognitive processes implicated in the maintenance of depression: rumination and overgeneralization. Rumination is repetitive thinking about the causes, meanings and implications of symptoms, problems and upsetting events (Nolen-Hoeksema, 1991; Watkins, 2008). Overgeneralization occurs when a negative general rule or abstract conclusion is drawn on the basis of isolated incidents and applied across the board to related and unrelated situations, for example a single negative event is interpreted as indicating a global, characterological inadequacy (Beck, 1976; Carver \& Ganellen, 1983). Both prospectively predict depressive symptoms (Carver, 1998; NolenHoeksema, 2000). Rumination can be adaptive, when characterized by a concrete and specific thinking style, or maladaptive, when characterized by an abstract and evaluative thinking style, with this latter style contributing to overgeneralization and depression (Watkins, 2008). These different thinking styles can be experimentally trained and causally influence emotional reactivity to a subsequent negative experience, with concrete training resulting in less depressed mood than abstract training (Watkins et al. 2008).

As a first step in translating this theoretical and experimental work into a viable intervention, Watkins et al. (2009) found that repeated CBM training to adopt the concrete style to negative experiences daily for a week (concreteness training, CNT) significantly reduced depression and rumination in dysphoric individuals, relative to no-training and attention controls, providing proof of principle that the abstract style causally contributes to the maintenance of depression. However, Watkins et al. (2009) (a) only examined training over 1 week, leaving unresolved whether benefits are maintained longer term; $(b)$ did not assess whether CNT changed diagnostic status for major depression; and (c) did not assess or account for current treatment as usual (TAU). Thus, the next step in developing and evaluating CNT as a viable intervention was to conduct a Phase II RCT (MRC, 2008).

Our primary objective was to assess the relative efficacy of CNT as a guided self-help intervention for patients with major depression in primary care within the National Health Service (NHS), relative to TAU. Our key hypothesis was that CNT adds benefit to TAU in reducing depression. To test this hypothesis, our study design and analysis plan stipulated a planned comparison between participants randomly allocated to TAU alone versus TAU plus CNT. This planned comparison minimizes potential threats to internal validity when examining the efficacy of CNT (e.g. maturation, spontaneous remission, repeated testing, regression to the mean).

A secondary objective was to explore the mechanism of CNT by examining the hypothesis that repeated practice at the concrete thinking exercises was a specific and active component of CNT that directly targeted rumination and overgeneralization. To explore whether CNT worked through specific processes over and above common therapy factors (e.g. rationale, therapist contact, amount and mode of practice) required a comparison with a complete treatment with common factor controls (Stevens et al. 2000). Therefore, our design and analysis plan included a planned comparison between participants randomly allocated to TAU plus either CNT or a control treatment matched for common and structural factors, as recommended by Baskin et al. (2003), but using an alternative noncognitive form of training. We selected progressive relaxation because $(a)$ it plausibly matches CNT for treatment rationale and affords plausible matched and repeated practice, unlike any inert attention control; (b) it is a non-cognitive treatment and there was no a priori theoretical reason to expect it to reduce rumination and overgeneralization. CNT significantly reducing depression relative to relaxation training (RT) would be one line of evidence supporting the hypothesis that the training exercises specific to CNT contribute to its treatment effects above and beyond common factors.

RT is not simply an attention control but rather an active evidence-based treatment demonstrated to 
reduce depression (Reynolds \& Coats, 1986; Murphy et al. 1995; Jorm et al. 2008). Moreover, it is difficult to demonstrate differential outcomes between two structurally matched treatments (Baskin et al. 2003). Thus, given the potential for no significant difference in outcomes between RT and CNT, we also explored the hypothesis that CNT was acting through different specific mechanisms rather than only common factors by investigating whether the matched treatments had differential effects on process measures. We predicted that CNT would significantly reduce rumination and overgeneralization relative to RT.

Furthermore, we hypothesized that CNT works when concrete thinking becomes habitual from repeated practice, such that it replaces the pre-existing habit of abstract thinking about difficulties that underlies rumination and overgeneralization (Watkins, 2008). We therefore expected CNT to be more efficacious when concrete thinking became habitual. As we hypothesized that RT does not act through changing habitual thinking style, we predicted greater treatment benefit for CNT relative to RT when the selfhelp responses (relaxation versus concreteness) had become habitual through repeated practice.

\section{Method}

\section{Design}

Participants were randomly allocated $1: 1: 1$ to TAU versus TAU $+\mathrm{CNT}$ versus $\mathrm{TAU}+\mathrm{RT}$. Block randomization was performed by an off-site, independent randomization service using computer-generated random codes stratified according to antidepressant use [the National Institute for Health and Clinical Excellence (NICE) recommended dose versus not receiving antidepressant/taking a subclinical dose] and severity of depression [mild to moderate depression, Hamilton Rating Scale for Depression (HAMD) score $\leqslant 17$, versus moderate to severe depression, HAMD score >17]. Patients were assessed by research staff blind to treatment allocation at intake baseline assessment and 8 weeks later (post-treatment) with faceto-face interviews, and at subsequent 3- and 6-month follow-ups (5 months, 8 months post-randomization) with telephone-based interviews. Fidelity of blinding was monitored: if a researcher was unblinded, an alternate researcher blind to allocation conducted subsequent assessments. The trial has been registered retrospectively (ISRCTN39455344).

\section{Participants}

Computerized databases in 15 primary care practices serving a population of around 300000 people across
Devon were searched to identify patients who had been prescribed antidepressant medication or recorded as depressed on Read codes for the previous 6 months. General practitioners (GPs) screened the list of eligible participants and wrote a letter to potential participants describing the study, enclosing the information sheet and inviting them to participate. Individuals who responded expressing interest in participating were contacted by the research team (principally by telephone) to discuss the study, screen for current depression using the Patient Health Questionnaire (PHQ-9), and if eligible, arrange a faceto-face baseline interview assessment. All participants gave signed written informed consent to participate.

Inclusion criteria were: age $\geqslant 18$ years, meeting DSM-IV criteria for a current episode of major depression $(n=105)$ or subthreshold $(n=16$, meeting four symptom criteria for major depression, at least one prolonged sad mood or loss of interest/pleasure, reflecting evidence that patients typically alternate between major depression and subthreshold symptoms ${ }^{1} \uparrow$, and that subthreshold symptoms predict a similar course to major depression; Judd et al. 1998; Fergusson et al. 2005), on the Structured Clinical Interview for DSM-IV (SCID; Spitzer et al. 1996) administered by a trained research worker (blind rating of randomly selected recorded interviews indicated excellent inter-rater reliability for major depression, $\kappa=0.9)$. Participants prescribed antidepressant medication needed to be taking a consistent dose for $\geqslant 4$ weeks before study entry to reduce the likelihood that recent medication change could account for symptom improvement. Exclusion criteria were: a history of bipolar disorder, psychosis, current substance/alcohol dependence, learning disability, organic brain damage, and concurrent psychotherapy at study entry. There were no exclusion criteria with respect to other Axis I or II diagnoses.

The trial CONSORT flowchart (Fig. 1) describes the flow of potential participants screened for eligibility through to randomization either to TAU +CNT $(n=40)$, TAU + RT $(n=39)$ or TAU $(n=42)$. The study was approved by the UK NHS North and East Devon Research Ethics Committee.

\section{Treatment conditions}

Both CNT and RT consisted of: (a) an initial individual face-to-face session lasting approximately $1.5 \mathrm{~h}$; (b) the patient practising the training exercises recorded on audiotape/compact disc (CD), supported by a detailed workbook, with a recommended frequency of 15-30 min daily for at least 6 weeks; (c) up

\footnotetext{
$\dagger$ The notes appear after the main text.
} 


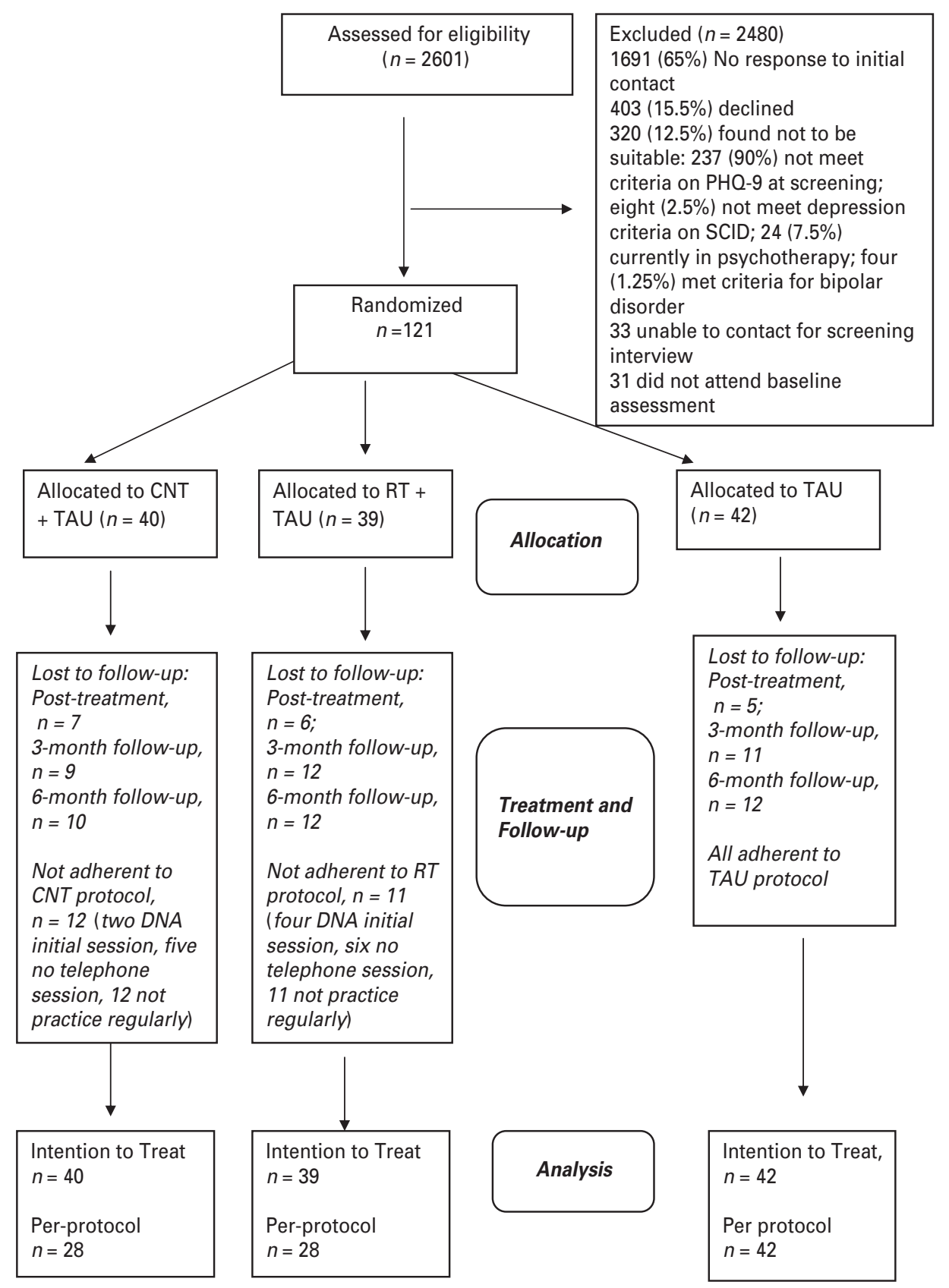

Fig. 1. CONSORT flow diagram. PHQ-9, Patient Health Questionnaire 9; CNT, concrete training guided self-help; RT, relaxation training guided self-help; TAU, treatment as usual.

to three 30-min telephone sessions, scheduled 1 week after the initial training and then at 2-week intervals. During the initial session, the trainer explained the treatment rationale, provided psycho-education about depression, rumination and overgeneralization, and practised training exercises with the patient. During the telephone sessions, the trainer provided feedback, guidance and encouragement to ensure accurate use of the exercises, monitored progress and scheduled regular practice. Training was provided by two graduate-level psychologists, two postdoctoral psychologists and one clinical psychologist, who used scripted manuals for each treatment. Trainers received weekly supervision to ensure therapy adherence.

In $\mathrm{CNT}$, the training exercises involved patients' identifying a recent mildly to moderately upsetting difficulty and working through standardized steps to facilitate concrete thinking: (i) using mental imagery to focus on sensory details during the difficult event, noticing what is specific about the event and the 
context in which it occurs; (ii) noticing the process and sequence by which the difficult event unfolds ('How did it happen?'), including warning signs and actions that may have influenced its outcome; (iii) focusing on how to move forward by specifying the particular steps and behaviours to do next (Watkins, 2009; Watkins et al. 2009). The practice CD included (a) $30 \mathrm{~min}$ repeating the original training exercise; $(b)$ a 7-min First Aid exercise in which concrete thinking is applied to difficulties in real time as they occur (practised in the first telephone session); (c) a 7-min 'absorption exercise' in which concrete thinking is used to enhance positive experiences (practised in the second telephone session).

In RT, the training exercises involved progressive relaxation skills including tensing and relaxing muscle groups and slowing breathing. The practice CD included (a) a 30-min progressive relaxation exercise; (b) a 7-min First Aid exercise using relaxation; (c) a 7-min exercise in which patients practised letting go of tension without prior tensing of muscles.

For all participants, TAU was current treatment provided by their primary care GP, including watchful waiting, regular appointments (across all conditions, mean 0.20 visits, S.D. $=0.40$, during the 8 -week treatment period), ongoing antidepressant medication and clinical management.

\section{Measures}

Our primary outcome was depressive symptoms assessed on the 17-item interviewer-rated HAMD (Hamilton, 1960; Williams, 1998), the most commonly used interview-based measure of depressive severity, with documented reliability and validity (range $0-52$; cut-offs: minimal depression $<8$, high severity $\geqslant 20$ ). Secondary outcomes included two well-validated and standardized self-report depression measures, the Beck Depression Inventory-II (BDI-II; Beck et al. 1996, range 0-63; cut-offs: 0-13 minimal, 14-19 mild, 20-28 moderate, 29-63 severe) and the PHQ-9 (Kroenke et al. 2001, range 0-27; cut-offs: 0-4 minimal, 5-10 mild, 11-15 moderate, 16-20 moderately severe, 20-27 severe), and a well-validated anxiety measure, the Generalized Anxiety Disorder-7 (GAD-7; Spitzer et al. 2006). A clinical psychologist experienced in the HAMD trained all research staff. Blind rating of randomly selected recorded interviews indicated excellent inter-rater reliability, $r=0.97$.

At baseline and post-treatment, patients completed the 22-item Ruminative Response Scale of the Response Styles Questionnaire (RSQ; Nolen-Hoeksema \& Morrow, 1991), the standard measure of depressive rumination, and a shortened version of the Attributional Style Questionnaire (ASQ; Peterson et al. 1982), which consisted of four hypothetical situations (two positive, two negative) to assess negative overgeneralization. Patients generated a description of the major cause for each event and rated the extent to which it was internal (due to self versus circumstances/others), stable (present in the future versus not) and global (influences many situations versus just this one). Negative overgeneralization was indexed by the composite score indicating the extent to which a negative event was rated as internal, stable and global. In addition, consistent with Watkins et al. (2009), an observer-rated measure of concreteness was obtained by a rater blind to condition scoring the patientgenerated causal descriptions of negative events on a $1-5$-point Likert scale $(1=$ abstract to $5=$ concrete) . There was good inter-rater reliability with a second blind rater $(r=0.89)$.

Where appropriate, patients completed the Morisky Medication Adherence instrument, a structured fouritem self-reported measure (scored $0-4,0-1=$ good adherence; $\geqslant 2$ signals potential difficulties with adherence), with demonstrated reliability and validity (Morisky et al. 1986), to assess adherence with antidepressant medication. Participants in CNT and RT completed the Credibility Expectancy Questionnaire (CEQ; Devilly \& Borkovec, 2000) to compare these treatments on perceived treatment expectancy and rationale credibility (ratings from 1 to 9 , higher score reflecting greater expectancy/credibility). During each telephone guided self-help session, patients reported the frequency and duration of their self-help practice, plus a single-item indexing whether the self-help response was becoming a habit $\left(1={ }^{\prime}\right.$ yes', $0=$ 'no' or 'unsure').

\section{Analysis}

The trial is reported in accord with the updated CONSORT guidelines for parallel group RCTs (Schulz et al. 2010). A direct estimate of the relative treatment effect of CNT versus TAU or RT was unavailable, so we used the effect size of CNT versus attention control for HAMD (Cohen's $d=0.91$ ) from Watkins et al. (2009) as a proxy. At an $\alpha$ of $5 \%$ and power of $90 \%$ it was estimated that 27 patients were required per treatment arm to demonstrate superiority of CNT over TAU. Assuming drop-out of $20-35 \%$, this indicates a total number for randomization of 100-125. Treatments are individualized so there is no need to inflate the standard error of the treatment comparison for potential clustering (Baldwin et al. 2005). Distinct analyses were conducted according to a predefined analysis plan. First, to test our primary hypothesis with regard to the effectiveness of CNT, we compared CNT with TAU + CNT. Second, although not formally powered, we 
compared TAU + CNT with TAU + RT on both outcome and process measures to explore whether CNT worked through specific CBM elements relative to common factors shared with RT.

The primary analyses involved intention-to-treat (ITT) and per protocol (PP) comparison of conditions at 8 weeks post-randomization adjusting for baseline outcome values and the stratification variables. ITT incorporated all patients according to and included in random allocation. The PP data set was defined as patients who stayed within stipulated key treatment parameters: for guided self-help, completing the initial training session, at least one telephone session, and practising the $\mathrm{CD}$ exercises four times a week for at least 2 weeks; for TAU, all patients were included. Secondary (ITT, PP) analyses compared primary and secondary outcomes between all treatment conditions at all follow-up points using a repeated-measures approach. All analytic models used an analysis of covariance (ANCOVA) regression approach, adjusting for baseline outcome values and the stratification variables. The effect size between conditions for change in outcome values from baseline to 8 weeks postrandomization was calculated using Cohen's $d$, where $d=$ (mean change for treatment condition $_{1}-$ mean change for treatment condition $\left.{ }_{2}\right) / \sigma_{\text {pooled, where }}$ $\sigma_{\text {pooled }}=\sqrt{ }\left[\left(\sigma_{1}^{2}+\sigma_{2}^{2}\right) / 2\right]$. Large effect sizes are defined as $\geqslant 0.80$.

For cases with missing data on the primary outcome, we used (i) last observation carried forward (which assumes data are missing at random) and (ii) regression analyses assuming data missing not at random to impute missing data (Choi \& Lu, 1995). Sensitivity analyses were undertaken to explore the impact of imputation of data losses on primary outcome analyses, comparing the results of the two imputation methods with a complete case analysis (imputed analyses are available from the authors). Analyses were unaffected by data imputation, and therefore we report the findings from the complete case analysis. All analyses were undertaken using SPSS version 16 (SPSS Inc., USA) and Stata version 10 (Stata Corporation, USA).

\section{Results}

\section{Patient flow and attrition}

Fig. 1 shows the patient flow from screening to follow-up including treatment adherence. In total, 121 patients met the study's criteria and agreed to participate. The overall attrition rate was $14.9 \%$ at posttreatment, $26.4 \%$ at the 3-month follow-up and $28.1 \%$ at the 6-month follow-up. There were no differences in rates of attrition across treatment condition. No patients reported dropping out because of adverse effects or dissatisfaction with treatment. Before the 3-month follow-up, one patient in TAU committed suicide. No further adverse events were adjudicated by the Trial Steering Committee.

There was no difference between TAU $+\mathrm{CNT}$ and TAU + RT for percentage of participants defined to be PP [CNT 70\%, RT 71.8\%, $\left.\chi^{2}(1 \mathrm{df})=0, p=1.00\right]$. In both conditions, patients within PP were comparable to patients outside PP on all characteristics at intake (all $p>0.10$ ). In TAU + CNT, patients within PP had significantly better outcomes than those outside PP for HAMD post-treatment $[F(1,30)=6.64, p=0.02$, PP mean $=7.40$, S.D. $=7.34$; not PP mean $=15.50$, S.D. $=$ 8.93]. In TAU + RT, the within and outside protocol RT groups were comparable on outcomes for HAMD post-treatment $(F<1.00)$.

\section{Baseline patient characteristics}

Table 1 shows patient characteristics of the ITT sample. There were no differences between treatment conditions on any of the patient characteristics, including antidepressant medication use and adherence. The principal antidepressants prescribed were selective serotonergic reuptake inhibitors (75\%), serotonergic-noradrenergic reuptake inhibitors (11.7\%) and tricyclic antidepressants $(8.3 \%)$.

CNT and RT did not differ for treatment credibility $[F(1,69)=2.41, p=0.12]$, treatment expectancy $(F<1)$ or attitude to the training following the initial session (1 not at all helpful; 7 extremely helpful) $(F<1.00)$, with both receiving positive endorsements.

\section{Primary analysis: clinical outcomes at 8 weeks}

Table 2 reports means and confidence intervals (CIs) for the primary and secondary outcome measures at the post-treatment assessment 8 weeks after randomization. With respect to our primary question of treatment efficacy, for both ITT and PP analyses, relative to TAU, TAU +CNT resulted in significantly fewer depressive symptoms post-treatment on the primary outcome HAMD (ITT, $p=0.006$, effect size $d$ for change in HAMD $=0.76 ; \mathrm{PP}, p<0.0001, d=1.06$ ), BDI-II (ITT, $p<0.0001, d=1.07$; PP $, p<0.0001, d=1.53)$ and PHQ-9 (ITT, $p<0.0001, d=0.89$; PP, $p<0.0001, d=1.17$ ) and significantly fewer anxious symptoms on the GAD-7 (ITT, $p=0.0001, d=0.85 ; \mathrm{PP}, p<0.0001, d=1.14$ ). There was no difference in reduction of symptoms of depression or anxiety for TAU $+\mathrm{CNT}$ compared to TAU + RT across all outcome measures, for ITT and PP analyses (all $p$ 's $>0.05$, except for PP analysis of PHQ-9 post-treatment, $p=0.04$ ).

Two exploratory subgroup analyses were undertaken to examine whether there was any interaction 
Table 1. Demographic and psychiatric characteristics of TAU+CNT, TAU $+R T$ and TAU intention-to-treat (ITT) sample at baseline

\begin{tabular}{|c|c|c|c|}
\hline & $\mathrm{TAU}+\mathrm{CNT}(n=40)$ & $\mathrm{TAU}+\mathrm{RT}(n=39)$ & TAU $(n=42)$ \\
\hline \multicolumn{4}{|l|}{ Demographic characteristics } \\
\hline Gender: female, $n(\%)$ & $26(65)$ & $29(74.4)$ & $23(54.8)$ \\
\hline Ethnicity: Caucasian, $n(\%)$ & $40(100)$ & $38(97.4)$ & $42(100)$ \\
\hline Age (years), mean (s.D.) & $46.37(12.71)$ & $46.05(11.60)$ & $46.38(12.30)$ \\
\hline Married/cohabiting, $\%$ & 48.7 & 69.2 & 60.97 \\
\hline Employed, \% & 62.16 & 66.66 & 59.10 \\
\hline \multicolumn{4}{|l|}{ Level of education, $n(\%)$} \\
\hline No educational qualifications & $6(16.2)$ & $6(16.2)$ & $6(14.3)$ \\
\hline Some school qualifications & $6(16.2)$ & $10(27.0)$ & $9(21.4)$ \\
\hline High school/vocational qualification & $21(56.8)$ & $16(43.2)$ & $19(45.2)$ \\
\hline University degree/professional qualification & $4(10.8)$ & $5(13.5)$ & $8(19.0)$ \\
\hline \multicolumn{4}{|l|}{ Psychiatric characteristics } \\
\hline HAMD, mean (S.D.) & $17.10(6.20)$ & $17.15(4.13)$ & $16.12(4.99)$ \\
\hline BDI-II, mean (S.D.) & $32.90(10.03)$ & $32.16(9.65)$ & $32.52(9.68)$ \\
\hline PHQ-9, mean (S.D.) & $17.82(4.28)$ & $17.77(4.01)$ & $17.36(3.79)$ \\
\hline GAD-7, mean (S.D.) & $12.57(5.22)$ & $14.74(4.80)$ & $12.38(4.76)$ \\
\hline ASQ-N, mean (S.D.) & $10.18(2.22)$ & $10.03(2.27)$ & $9.73(2.49)$ \\
\hline O-R CN, mean (S.D.) & $2.40(0.69)$ & $2.53(0.94)$ & $2.48(0.93)$ \\
\hline RSQ, mean (S.D.) & $55.61(10.22)$ & $56.97(11.65)$ & $56.32(11.17)$ \\
\hline No. of prior episodes, mean (S.D.) & $4.13(3.17)$ & $4.46(3.40)$ & $5.00(3.85)$ \\
\hline Duration of current episode (months), mean (s.D.) & $27.73(42.60)$ & $36.63(67.79)$ & $23.17(41.94)$ \\
\hline Age of onset (years), mean (s.D.) & $29.22(13.89)$ & $25.62(12.51)$ & $26.15(13.42)$ \\
\hline Prior psychiatric hospitalization, $n(\%)$ & $3(7.5)$ & $8(20.5)$ & $7(16.7)$ \\
\hline Prior suicide attempt, $n(\%)$ & $12(30)$ & $10(25.6)$ & $11(26.2)$ \\
\hline Any Axis I co-morbidity, $n(\%)$ & $27(67.5)$ & $31(79.5)$ & $30(71.4)$ \\
\hline No. co-morbid Axis I diagnoses, mean (S.D.) & $1.37(1.27)$ & $1.77(1.40)$ & $1.45(1.38)$ \\
\hline Co-morbid anxiety disorders, $n(\%)$ & $26(65)$ & $30(76.9)$ & $26(61.9)$ \\
\hline Antidepressant medication use, $\%$ & 47.5 & 43.6 & 50 \\
\hline Antidepressant adherence, mean (s.D.) & $1.11(1.08)$ & $1.06(1.21)$ & $1.25(1.03)$ \\
\hline Treatment credibility & $6.97(1.17)$ & $6.53(1.21)$ & N.A. \\
\hline Treatment expectancy & $6.17(1.77)$ & $5.81(1.55)$ & N.A. \\
\hline Attitude to training & $6.06(1.04)$ & $6.00(0.92)$ & N.A. \\
\hline
\end{tabular}

TAU, Treatment-as-usual; CNT, concrete training guided self-help; RT, relaxation training guided self-help ;

HAMD, Hamilton Rating Scale for Depression; BDI-II, Beck Depression Inventory-II; PHQ-9, Patient Health Questionnaire-9 ; ASQ-N, Attributional Style Questionnaire composite score for negative events; O-R CN, observer-rated score for concreteness of patient-generated description of causes for negative events on ASQ; RSQ, rumination scale of the Response Style Questionnaire ; S.D., standard deviation; N.A., not applicable.

effect of the stratification variables on the primary outcome at 8 weeks. There was no evidence of a differential response to treatments for those prescribed antidepressants versus those not prescribed antidepressants (TAU + CNT $v$. TAU $: p=0.37$; TAU + CNT $v$. TAU + RT: $p=0.23)$. However, there was evidence of a significant interaction $(p=0.02)$ between treatment condition (TAU + CNT, TAU) and stratification by baseline HAMD severity, reflecting a greater differential treatment effect between TAU +CNT and TAU at mild to moderate levels of depression. No significant interaction effect $(p=0.23)$ for baseline HAMD was seen for TAU $+\mathrm{CNT}$ versus $\mathrm{TAU}+\mathrm{RT}$.

\section{Primary analyses: processes of therapy}

At post-treatment follow-up, TAU $+\mathrm{CNT}$ resulted in a significantly greater reduction in rumination (ITT, $p<0.001$; $\mathrm{PP}, \quad p<0.0001)$ and negative overgeneralization (ITT, $p=0.07$; PP, $p=0.012$ ), and a significantly greater increase in observer-rated concreteness for patient-generated problem descriptions (ITT, $p=0.006$; PP, $p=0.01$ ) than TAU (see Table 2). As predicted by the hypothesis that CNT specifically targets these cognitive processes, TAU $+\mathrm{CNT}$ produced a greater reduction in rumination (ITT, $p=0.006$; PP, $p=0.004)$ and negative overgeneralization (ITT, $p=$ $0.03 ; \mathrm{PP}, p=0.02)$, and a significantly greater increase 
Table 2. Primary analysis: comparison of outcomes at 8 weeks

\begin{tabular}{|c|c|c|c|c|c|}
\hline \multirow{3}{*}{ Condition } & \multicolumn{3}{|c|}{8 weeks (mean, s.D.) } & \multirow{3}{*}{$\begin{array}{l}\begin{array}{l}\text { Mean difference } \\
(95 \% \mathrm{CI}), p \text { value }\end{array} \\
\text { TAU-TAU }+\mathrm{CNT}\end{array}$} & \multirow{3}{*}{$\begin{array}{l}\text { Mean difference } \\
(95 \% \mathrm{CI}), p \text { value } \\
(\mathrm{TAU}+\mathrm{RT})-(\mathrm{TAU}+\mathrm{CNT})\end{array}$} \\
\hline & \multicolumn{3}{|c|}{ Intention-to-treat (ITT) analysis } & & \\
\hline & $(n=33)$ & $(n=33)$ & $(n=37)$ & & \\
\hline HAMD & $9.36(8.39)$ & $11.33(7.20)$ & $13.00(6.25)$ & $4.28(1.29-7.26), 0.006$ & $1.98(-1.14$ to 5.11$), 0.21$ \\
\hline BDI-II & $18.36(15.21)$ & $21.36(12.84)$ & $29.06(11.06)$ & $10.28(5.55-15.03),<0.0001$ & $3.20(-2.37$ to 8.78$), 0.26$ \\
\hline PHQ-9 & $8.75(7.70)$ & $10.84(7.13)$ & $14.76(6.13)$ & $5.95(2.81-9.08),<0.0001$ & $2.09(-0.93$ to 5.11$), 0.17$ \\
\hline GAD-7 & $6.12(6.11)$ & $8.45(4.90)$ & $10.56(5.66)$ & $4.14(1.86-6.43), 0.001$ & $0.73(-1.33$ to 2.80$), 0.48$ \\
\hline RSQ & $41.06(14.71)$ & $49.81(12.99)$ & $51.00(12.58)$ & 9.69 (4.00-15.37), 0.001 & $8.31(2.50-14.13), 0.006$ \\
\hline ASQ-N & $8.01(2.41)$ & $9.57(2.56)$ & $9.10(2.25)$ & $1.16(-0.11$ to 2.42$), 0.07$ & $1.39(0.15-2.64), 0.028$ \\
\hline \multirow[t]{3}{*}{ O-R CN } & $3.04(0.78)$ & $2.55(0.76)$ & $2.33(0.91)$ & $-0.72(-1.22$ to -0.22$), 0.006$ & $-0.50(-0.95$ to -0.06$), 0.027$ \\
\hline & \multicolumn{3}{|c|}{ Per protocol (PP) analysis } & & \\
\hline & $(n=25)$ & $(n=27)$ & $(n=37)$ & & \\
\hline HAMD & $7.40(7.35)$ & $11.19(7.60)$ & $13.00(6.25)$ & $5.92(2.81-9.03),<0.0001$ & $2.94(-0.43$ to 6.32$), 0.09$ \\
\hline BDI-II & $13.88(11.98)$ & $19.61(11.91)$ & $29.06(11.06)$ & $13.55(9.02-18.07),<0.0001$ & $4.37(-1.23$ to 9.96$), 0.12$ \\
\hline PHQ-9 & $6.33(5.68)$ & $10.40(7.36)$ & $14.76(6.13)$ & $8.01(4.92-11.11),<0.0001$ & $3.26(0.14-6.38), 0.04$ \\
\hline GAD-7 & $5.04(4.82)$ & $7.96(4.90)$ & $10.56(5.66)$ & $5.15(2.79-7.50),<0.0001$ & $1.00(-1.15$ to 3.16$), 0.35$ \\
\hline RSQ & $38.46(12.73)$ & $49.68(13.35)$ & $51.00(12.58)$ & $12.02(6.25-17.79),<0.0001$ & 9.68 (3.27-16.09), 0.004 \\
\hline ASQ-N & $7.50(2.14)$ & $9.53(2.79)$ & $9.10(2.25)$ & $1.66(0.39-2.94), 0.012$ & $1.70(0.335-3.07), 0.016$ \\
\hline $\mathrm{O}-\mathrm{R} \mathrm{CN}$ & $3.05(0.86)$ & $2.59(0.76)$ & $2.33(0.91)$ & $-0.73(-1.29$ to -0.16$), 0.013$ & $-0.46(-0.99$ to 0.07$), 0.09$ \\
\hline
\end{tabular}

TAU, Treatment-as-usual; CNT, concrete training guided self-help; RT, relaxation training guided self-help; CI, confidence interval; HAMD, Hamilton Rating Scale for Depression; BDI-II, Beck Depression Inventory-II; PHQ-9, Patient Health Questionnaire-9; GAD-7, Generalized Anxiety Disorder-7; RSQ, ruminative scale of Response Styles Questionnaire; ASQ-N, Attributional Style Questionnaire composite score for negative events; O-R CN, observer-rated score for concreteness of patientgenerated description of causes for negative events on the ASQ.

${ }^{a}$ Adjusted for baseline score and stratification variables for completers.

in observer-rated concreteness (ITT, $p=0.03$; PP, $p=0.09)$ than TAU + RT.

To test the hypothesis that CNT is effective when the concrete thinking style becomes habitual, we examined whether the self-help response being habitual at the final telephone session interacted with treatment condition to influence primary outcome at 8 weeks. As hypothesized, there was evidence of a significant interaction $(p=0.02)$ between treatment condition and report of habitual use of self-help, reflecting TAU + CNT having a greater treatment effect than TAU + RT when the self-help response became habitual ( $p=0.02,95 \%$ CI for mean benefit of CNT 0.63-7.91), but the reverse pattern when it did not $(p=0.06,95 \% \mathrm{CI}$ for mean benefit of CNT -10.54 to 0.33).

\section{Secondary analyses}

Figs 2 and 3 show the symptoms of depression for each treatment condition across the four assessment points for ITT and PP respectively ${ }^{2}$. When comparing $\mathrm{TAU}+\mathrm{CNT}$ versus TAU in the repeated-measures analysis, there was a trend towards a significant main effect of condition on the HAMD [ITT, $F(1,47)=2.61$, $p=0.11$; PP, $F(1,43)=3.88, p=0.06]$, which was secondary to a significant interaction of condition by follow-up [ITT, $F(2,46)=5.11, p=0.01$; PP, $F(2,42)=$ 5.94, $p=0.005]$. Decomposing this interaction, the significantly greater reduction on the HAMD for $\mathrm{TAU}+\mathrm{CNT}$ compared to TAU at post-treatment was no longer significant at the 3-month follow-up [ITT, $F(4,57)=0.25, p=0.62 ; \mathrm{PP}, F(4,49)=0.007, p=0.94]$, but was borderline significant at the 6-month followup for PP analysis $[F(4,48)=4.05, p=0.05]$ but not for ITT analysis $[F(4,55)=1.93, p=0.17]$. On the BDI, there was a significant main effect of condition [ITT, $F(1,36)=8.65, p=0.006$; PP, $F(1,34)=10.16, p=0.003$ ], and no interaction of condition by follow-up ( $F^{\prime} \mathrm{s}$ $<1.00$ ), reflecting lower depressive symptoms across all three follow-ups for TAU + CNT compared to TAU. 

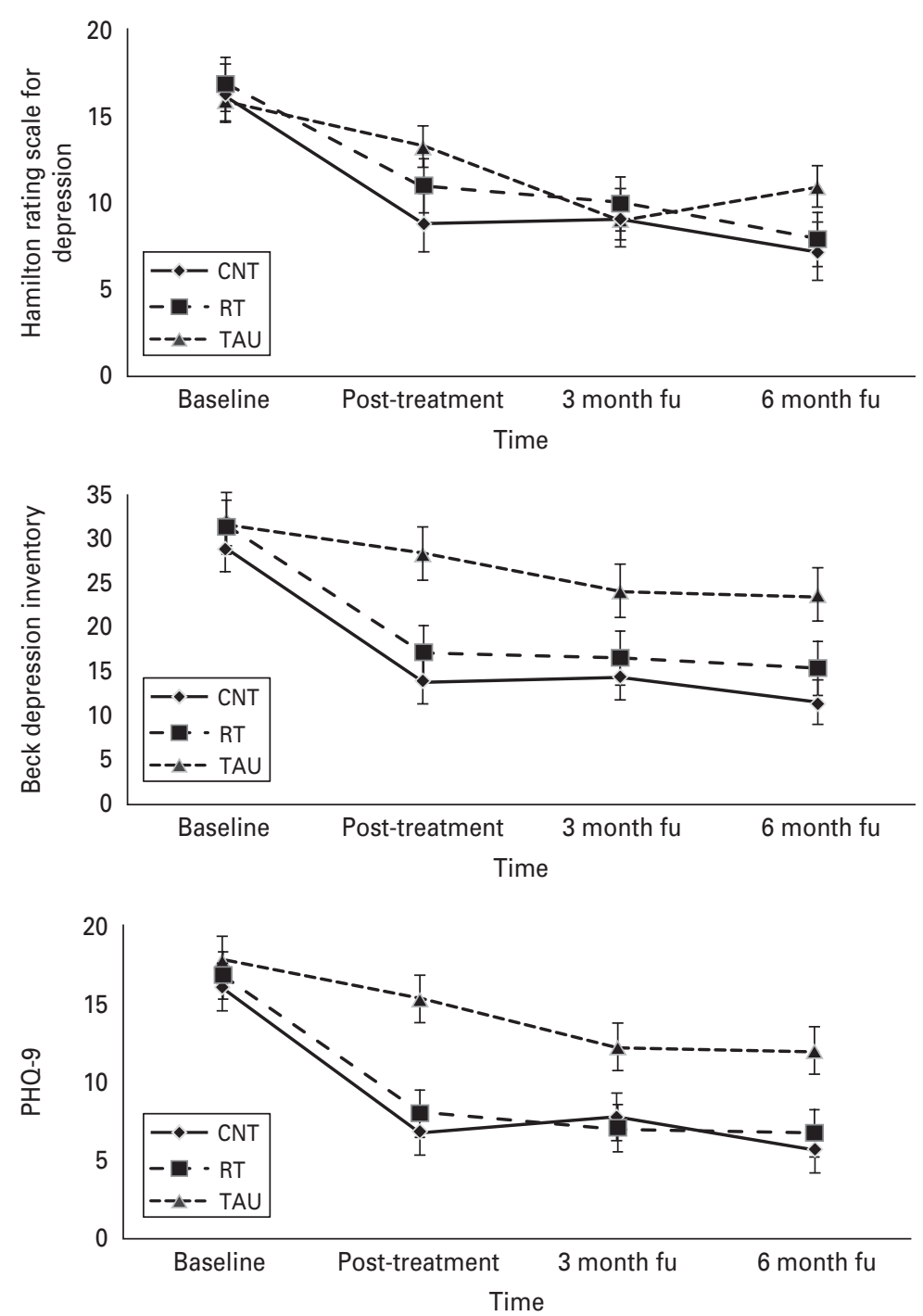

Fig. 2. Depressive symptoms at baseline and follow-up assessments by treatment condition [intention-to-treat (ITT) analysis on case completers] : CNT, concrete training guided self-help; RT, relaxation training guided self-help; TAU, treatment as usual; $\mathrm{fu}$, follow-up.

Likewise on the PHQ-9, there was a significant main effect of condition [ITT, $F(1,37)=7.51, p=0.009$; PP, $F(1,35)=9.51, p=0.004]$ and no interaction of condition by follow-up [ITT, $F(2,36)=2.33, p=0.11$; PP, $F(2,34)=2.25, p=0.12]$. On the GAD-7, there was a significant main effect of condition [ITT, $F(1,37)=7.30$, $p=0.01$; PP, $F(1,35)=9.03, p=0.005]$ and no interaction of condition by follow-up [ITT, $F(2,36)=2.17$, $p=0.13$; PP, $F(2,34)=2.82, p=0.07$ ], reflecting lower anxiety for TAU + CNT compared to TAU across all three follow-ups.

When comparing TAU $+\mathrm{CNT}$ versus $\mathrm{TAU}+\mathrm{RT}$, there was no significant main effect of condition (all $F^{\prime} \mathrm{s}<1.00$ ) or interaction of condition by follow-up on all outcome measures (all $F^{\prime} \mathrm{s}<1.00$ ) for both ITT and PP analyses.

\section{Discussion}

With respect to the primary objective, the analyses indicate that CNT has added benefit relative to TAU in reducing depressive symptoms in patients with major depression in primary care, suggesting that CNT may be an efficacious treatment. These results extend the proof-of-principle findings indicating a benefit of CNT in dysphoric individuals (Watkins et al. 2009) to patients with major depression who are receiving treatment from their GP. Moreover, the benefits of CNT were stable and durable over time: the improvements in symptoms found post-intervention were maintained at the 3- and 6-month follow-ups. This differential treatment effect was only significant for mild to moderate levels of depression. 

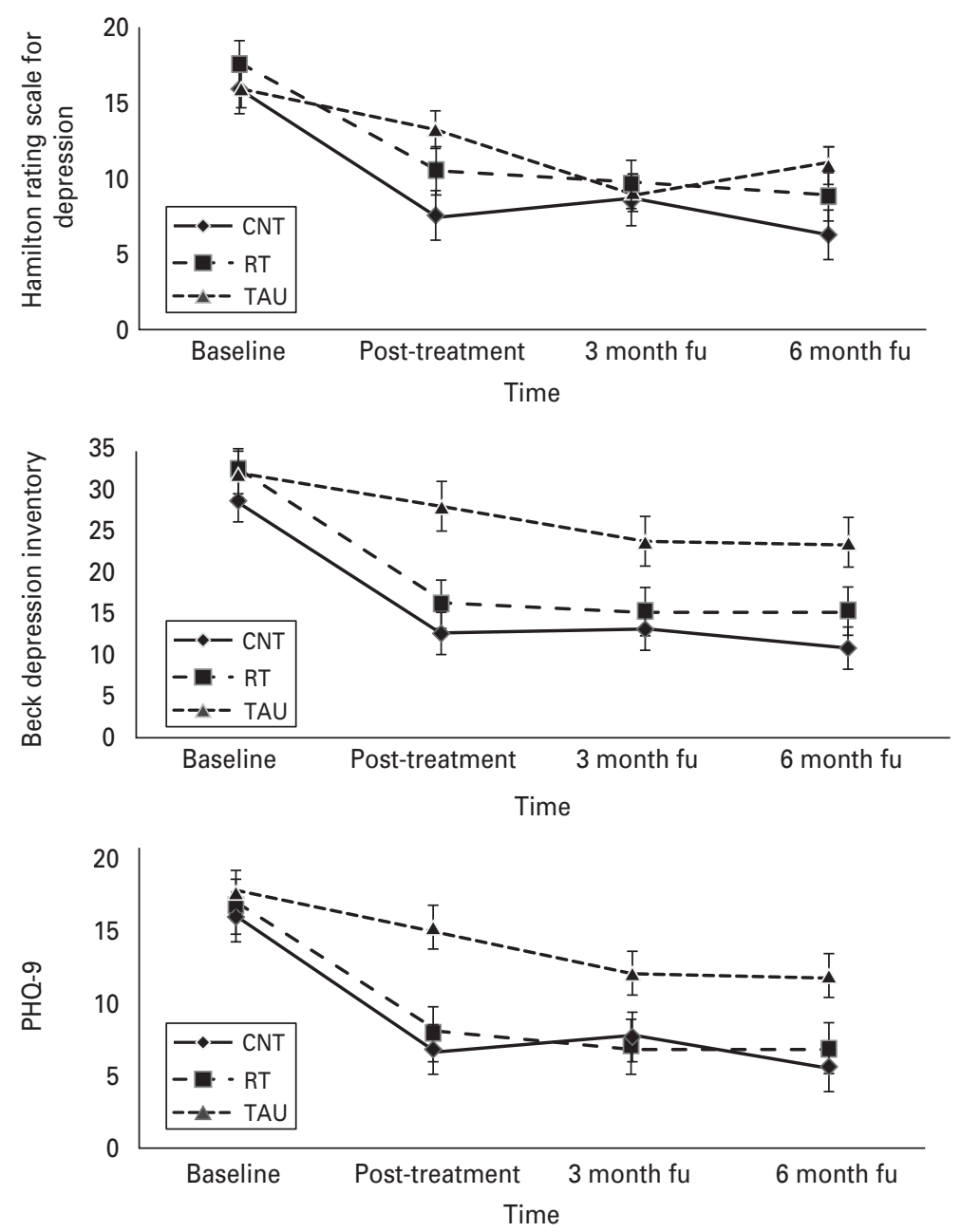

Fig. 3. Depressive symptoms at baseline and follow-up assessments by treatment condition [per protocol (PP) analysis on case completers]: CNT, concrete training guided self-help; RT, relaxation training guided self-help; TAU, treatment as usual ; $\mathrm{fu}$, follow-up.

The secondary objective was to explore the mechanism of how CNT works by comparing processes for CNT with RT, which was matched for common and structural factors, and only differed in using a non-cognitive training exercise (relaxation). Perhaps unsurprisingly, as the Phase II trial was not formally powered for this comparison, there was no difference between CNT and RT on any symptom measure at any time point. Our results indicate that these treatments are likely to be of similar efficacy. The most parsimonious interpretation is that the whole treatment effect of both therapies was due to common treatment factors including contact with a supportive trainer, a plausible rationale, and the provision of an active coping response. However, the absence of treatment differences does not necessarily imply that common factors account for all effects: an alternative is that CNT and RT worked through different active mechanisms. RT could have worked through reducing general tension, thereby facilitating improvement in associated depression symptoms and/or by enhancing perceived self-efficacy. Consistent with the possibility of different mechanisms, CNT reduced rumination and overgeneralization significantly more than RT post-intervention. Although this could reflect demand effects on these self-report measures, this seems unlikely because both treatments emphasized how they reduce rumination and overgeneralization and did not differ on credibility or treatment expectancy. Moreover, CNT increased concreteness of problem descriptions significantly more than RT and TAU on a performance-based non-self-report measure, consistent with Watkins et al. (2009). Finally, consistent with the hypothesis that CNT works through repeated concrete thinking establishing an alternative habitual response to replace rumination and overgeneralization, CNT reduced depression significantly more than RT post-intervention in those patients who 
reported that the practised self-help response had become habitual. Given the relatively small sample size for this interaction $(n=64)$, we need to be cautious about this provisional finding. Nonetheless, if replicated, it suggests the potential therapeutic importance of ensuring that adaptive cognitive responses (e.g. concreteness) become habitual so as to effectively reduce symptoms during CBM training. We tentatively suggest that these convergent findings provide preliminary evidence consistent with the hypothesis that CNT works through a specific effect of the concreteness exercises on shifting rumination and overgeneralization in a way distinct from RT. Nonetheless, the CNT paradigm would benefit from further development work to understand and enhance its active mechanism(s).

These findings also suggest that CNT is a feasible and acceptable treatment in primary care. Ratings of expectancy and credibility were high, and rates of drop-out were comparable with individual CBT (e.g. DeRubeis et al. 2005, 15\% at 8 weeks). The effect size of CNT relative to TAU post-treatment is large $(d=0.76-1.07)$ and comparable to that reported for guided self-help relative to waiting list and usual care/attention controls combined $(d=0.8,95 \% \mathrm{CI}$ 0.58-1.01; Gellatly et al. 2007). Moreover, the treatment benefits of CNT seemed to be maintained at the 3- and 6-month follow-ups. These findings indicate the potential of CNT as an intervention for depression in primary care, especially for mild to moderate levels of symptoms. As CNT only requires minimal therapist contact (approximately $3 \mathrm{~h}$ ), it may provide a costeffective way to make psychological treatments more widely available. Moreover, CBM training lends itself to automatization and delivery through information technology (computerized training, internet delivery, Smartphone apps), making it highly accessible. If these initial findings are confirmed, CNT has the potential to become a useful option within the expanding repertoire of treatments for common mental health problems.

This study has several strengths including (a) being powered for the primary comparison and an assumed level of attrition that was representative of actual patient flow; (b) a range of symptom measures; (c) well-operationalized and matched experimental and control treatments; $(d)$ randomized allocation to treatment arms; and (d) 3- and 6-month follow-ups. Limitations include imperfect uptake of the interventions, less than optimal power for comparison of CNT versus RT, and the risk of attrition bias due to loss to follow-up/drop-out. However, as results on the primary outcome were not sensitive to multiple methods of imputation, attrition bias is likely to be small.
In conclusion, this exploratory Phase II RCT indicates that an innovative form of cognitive training that involves direct practice at concrete thinking in response to difficulties seems to be an efficacious treatment for mild to moderate depression in primary care, producing significantly better outcomes than TAU. There is preliminary evidence indicating that CNT may specifically influence depressogenic cognitive processes such as rumination and overgeneralization. Nonetheless, as the first exploratory trial, there is a need for further RCTs to replicate these findings in other settings based on the extant effect sizes observed in this study and to examine cost-effectiveness in a fully powered Phase III trial.

\section{Acknowledgements}

This trial was funded by a Medical Research Council Experimental Medicine Grant to E.R. Watkins (GO502003 ID 77156). We are grateful to the patients who participated in the trial and to the physicians and other health-care staff who enabled the trial. The authors are listed in the following order: principal investigator, co-investigators, and research staff.

\section{Declaration of Interest}

None.

\section{Notes}

1 The pattern of results was the same when the sample was limited to those patients meeting full diagnostic criteria for major depression.

${ }^{2}$ At the 8-week post-treatment follow-up, there was no difference between the treatment conditions in antidepressant use $(p=0.43$; CNT $39.4 \%$, RT $51.5 \%$, TAU $54.0 \%$ ). At the 3- and 6-month follow-ups, there were trends towards differences in rates of antidepressant use $(p=0.076$ and 0.062 respectively), reflecting increasing antidepressant use in RT and TAU relative to CNT (3-month follow-up: CNT 38.7\%, RT $63.0 \%$, TAU $64.5 \%$; 6-month follow-up: CNT $38.7 \%$, RT 59.3\%, TAU 67.7\%).

\section{References}

Baldwin SA, Murray DM, Shadish WR (2005). Empirically supported treatments or type I errors? Problems with the analysis of data from group-administered treatments. Journal of Consulting and Clinical Psychology 73, 924-935.

Baskin TW, Tierney SC, Minami T, Wampold BE (2003). Establishing specificity in psychotherapy: a meta-analysis of structural equivalence of placebo controls. Journal of Consulting and Clinical Psychology 71, 973-979.

Beck AT (1976). Cognitive Therapy and Emotional Disorders. Meridian: New York. 
Beck AT, Steer RA, Brown GK (1996). The Beck Depression Inventory, Second Edition. The Psychological Corporation: San Antonio, TX.

Blazer DG, Kessler RC, McGonagle KA, Swartz MS (1994). The prevalence and distribution of major depression in a national community sample: the National Comorbidity Survey. American Journal of Psychiatry 151, 979-986.

Carver CS (1998). Generalization, adverse events, and development of depressive symptoms. Journal of Personality 66, 607-619.

Carver CS, Ganellen RJ (1983). Depression and components of self-punitiveness: high standards, self-criticism, and overgeneralization. Journal of Abnormal Psychology 92, 330-337.

Choi SC, Lu IL (1995). Effect of non-random missing data mechanisms in clinical trials. Statistical Medicine 14, 2675-2684.

Christensen H, Griffiths KM, Jorm AF (2004). Delivering interventions for depression by using the internet: randomised controlled trial. British Medical Journal 328, 265.

DeRubeis RJ, Hollon SD, Amsterdam JD, Shelton RC, Young PR, Saloman RM, O'Reardon JP, Lovett ML, Gladis MM, Brown LL, Gallop R (2005). Cognitive therapy versus medications in the treatment of moderate to severe depression. Archives of General Psychiatry 62, 409-416.

Devilly GJ, Borkovec TD (2000). Psychometric properties of the credibility/expectancy questionnaire. Journal of Behaviour Therapy and Experimental Psychiatry 31, 73-86.

Fergusson DM, Horwood LJ, Ridder EM, Beautrais AL (2005). Subthreshold depression in adolescence and mental health outcomes in adulthood. Archives of General Psychiatry 62, 66-72.

Gellatly J, Bower P, Hennessy S, Richards D, Gilbody S, Lovell K (2007). What makes self-help interventions effective in the management of depressive symptoms? Meta-analysis and meta-regression. Psychological Medicine 37, 1217-1228.

Hakamata Y, Lissek S, Bar-Haim Y, Britton JC, Fox NA, Leibenluft E, Ernst M, Pine DS (2010). Attention Bias Modification Treatment: a meta-analysis toward the establishment of a novel treatment for anxiety. Biological Psychiatry 68, 982-990.

Hamilton M (1960). A rating scale for depression. Journal of Neurology, Neurosurgery, and Psychiatry 12, 52-62.

Hollon SD, Munoz RF, Barlow DH, Beardslee WR, Bell CC, Bernal G, Clarke GN, Franciosi LP, Kazdin AE, Kohn L, Linehan MM, Markowitz JC, Miklowitz DJ, Persons JB, Niederehe G, Sommers D (2002). Psychosocial intervention development for the prevention and treatment of depression: promoting innovation and increasing access. Biological Psychiatry 52, 610-630.

Jorm AF, Morgan AJ, Hetrick SE (2008). Relaxation for depression. Cochrane Database of Systematic Reviews 4, CD007142.

Judd LL (1997). The clinical course of unipolar major depressive disorders. Archives of General Psychiatry 54, 989-991.
Judd LL, Akiskal HS, Maser JD, Zeller PJ, Endicott J, Coryell W, Paulus MP, Kunovac JL, Leon AC, Mueller TI, Rice JA, Keller MB (1998). A prospective 12-year study of subsyndromal and syndromal depressive symptoms in unipolar major depressive disorders. Archives of General Psychiatry 55, 694-700.

Kessler D, Lewis G, Kaur S, Wiles N, King M, Weich S, Sharp DJ, Araya R, Hollinghurst S, Peters TJ (2009). Therapist-delivered internet psychotherapy for depression in primary care: a randomized controlled trial. Lancet 374, 628-634.

Kroenke K, Spitzer RL, Williams JBW (2001). The PHQ-9: validity of a brief depression severity measure. Journal of General Internal Medicine 16, 606-613.

Layard R (2005). Mental health: Britain's biggest social problem? Paper presented at the No. 10 Strategy Unit Seminar on Mental Health on 20 January 2005 (http:/ / cep.lse.ac.uk/textonly/research/mentalhealth/ RL414d.pdf).

MacLeod C, Rutherford E, Campbell L, Ebsworthy G, Holker L (2002). Selective attention and emotional vulnerability: assessing the causal basis of their association through the experimental manipulation of attentional bias. Journal of Abnormal Psychology 111, 107-123.

Mathews A, MacLeod C (2002). Induced processing biases have causal effects on anxiety. Cognition and Emotion 16, 331-354.

Morisky DE, Green LW, Levine DM (1986). Concurrent and predictive validity of a self-reported measure of medication adherence. Medical Care 24, 67-74.

MRC (2008). Developing and Evaluating Complex Interventions: New Guidance (www.mrc.ac.uk/ complexinterventionsguidance) Accessed 10 November 2008.

Murphy GE, Carney RM, Knesevich MA, Wetzel RD, Whitworth P (1995). Cognitive behavior therapy, relaxation training, and tricyclic antidepressant medication in the treatment of depression. Psychological Reports 77, 403-420.

Nolen-Hoeksema S (1991). Responses to depression and their effects on the duration of depressive episodes. Journal of Abnormal Psychology 100, 569-582.

Nolen-Hoeksema S (2000). The role of rumination in depressive disorders and mixed anxiety/ depressive symptoms. Journal of Abnormal Psychology 109, 504-511.

Nolen-Hoeksema S, Morrow J (1991). A prospective study of depression and posttraumatic stress symptoms after a natural disaster: the 1989 Loma Prieta Earthquake. Journal of Personality and Social Psychology 61, 115-121.

Peterson C, Semmel A, von Baeyer C, Abramson LY, Metalsky GI, Seligman MEP (1982). The Attributional Style Questionnaire. Cognitive Therapy and Research 6, 287-300.

Reynolds WM, Coats KI (1986). A comparison of cognitive behavioral therapy and relaxation training for the treatment of depression in adolescents. Journal of Consulting and Clinical Psychology 54, 653-660. 
Schulz K, Altman DG, Moher D (2010). CONSORT 2010 statement: updated guidelines for reporting parallel group randomized trials. Annals of Internal Medicine 152, $1-6$.

Spitzer RL, Kroenke K, Williams JBW, Löwe B (2006). A brief measure for assessing generalized anxiety disorder: the GAD-7. Archives of Internal Medicine 166, 1092-1097.

Spitzer RL, Williams JBW, Gibbon M, First MB (1996). Structured Clinical Interview for DSM-IV (SCID). American Psychiatric Association: Washington, DC.

Stevens SE, Hynan MT, Allen M (2000). A meta-analysis of common factor and specific treatment effects across domains of the phase model of psychotherapy. Clinical Psychology: Science and Practice 7, 273-290.

Ustun TB, Ayuso-Mateos JL, Chatterji S, Mathers C, Murray CJL (2004). Global burden of depressive disorders in the year 2000. British Journal of Psychiatry 184, 386-392.

Watkins ER (2008). Constructive and unconstructive repetitive thought. Psychological Bulletin 134, 163-206.
Watkins ER (2009). Depressive rumination: investigating mechanisms to improve cognitive behavioural treatments. Cognitive Behaviour Therapy 38, 8-14.

Watkins ER, Baeyens CB, Read R (2009). Concreteness training reduces dysphoria: proof-of-principle for repeated cognitive bias modification in depression. Journal of Abnormal Psychology 118, 55-65.

Watkins ER, Moberly NJ, Moulds M (2008). Processing mode causally influences emotional reactivity: distinct effects of abstract versus concrete construal on emotional response. Emotion 8, 364-378.

Wells TT, Beevers CG (2010). Biased attention and dysphoria: manipulating selective attention reduces subsequent depressive symptoms. Cognition and Emotion 24, 719-728.

Williams JBW (1998). A structured clinical interview guide for the Hamilton Depression Rating Scale. Archives of General Psychiatry 45, 742-747.

Wulsin LR, Vaillant GE, Wells VE (2004). A systematic review of the mortality of depression. Psychosomatic Medicine 61, 6-17. 